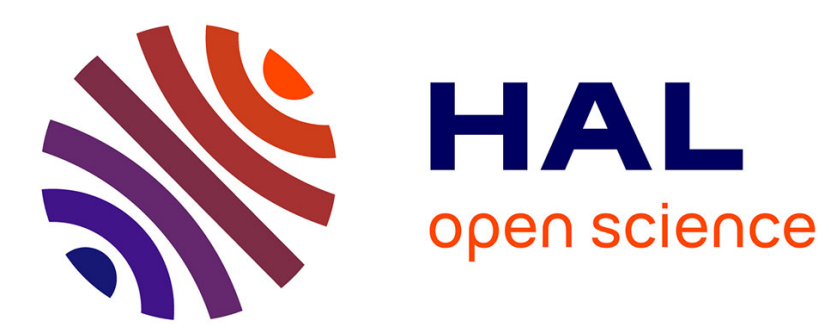

\title{
Meta-model-assisted MGDA for multi-objective functional optimization
}

Adrien Zerbinati, Andrea Minelli, Imane Ghazlane, Jean-Antoine Desideri

\section{To cite this version:}

Adrien Zerbinati, Andrea Minelli, Imane Ghazlane, Jean-Antoine Desideri. Meta-model-assisted MGDA for multi-objective functional optimization. Computers and Fluids, 2014, 102 (10), pp.116 - 130. 10.1016/j.compfluid.2014.06.018 . hal-01082595

\section{HAL Id: hal-01082595 https://hal.science/hal-01082595}

Submitted on 13 Nov 2014

HAL is a multi-disciplinary open access archive for the deposit and dissemination of scientific research documents, whether they are published or not. The documents may come from teaching and research institutions in France or abroad, or from public or private research centers.
L'archive ouverte pluridisciplinaire HAL, est destinée au dépôt et à la diffusion de documents scientifiques de niveau recherche, publiés ou non, émanant des établissements d'enseignement et de recherche français ou étrangers, des laboratoires publics ou privés. 


\title{
Meta-Model-Assisted MGDA for Multi-Objective Functional Optimization
}

\author{
Adrien Zerbinati ${ }^{\mathrm{a}}$, Andrea Minelli ${ }^{\mathrm{b}}$, Imane Ghazlane ${ }^{\mathrm{b}}$, Jean Antoine \\ Désidéri $^{\mathrm{a}}$ \\ ${ }^{a}$ OPALE Project Team, INRIA Sophia Antipolis Méditerranée, 06902, Sophia Antipolis, \\ France. \\ ${ }^{b}$ Department of Applied Aerodynamics, Civil Aircraft Unit, ONERA - The French \\ Aerospace Lab, F-92190, Meudon, France
}

\section{Abstract}

A novel numerical method for multi-objective differentiable optimization, the Multiple-Gradient Descent Algorithmm (MGDA), has been proposed in [8] [11] to identify Pareto fronts. In MGDA, a direction of search for which the directional gradients of the objective functions are all negative, and often equal by construction [12], is identified and used in a steepest-descent-type iteration. The method converges to Pareto-optimal points. MGDA is here briefly reviewed to outline its principal theoretical properties and applied first to a classical mathematical test-case for illustration. The method is then extended encompass cases where the functional gradients are approximated via meta-models, as it is often the case in complex situations, and demonstrated on three optimum-shape design problems in compressible aerodynamics. The first problem is purely related to aerodynamic performance. It is a wing shape optimization exercise w.r.t. lift and drag in typical transonic cruise

Email addresses: adrien.zerbinati@inria.fr (Adrien Zerbinati), Andrea.Minelli@onera.fr (Andrea Minelli), Imane.Ghazlane@onera.fr (Imane Ghazlane), Jean-Antoine.Desideri@inria.fr (Jean Antoine Désidéri) 
conditions. The second problem involves the aerodynamic performance and an environmental criterion : a supersonic glider configuration is optimized w.r.t. drag under lift constraint concurrently with a measure of the sonicboom intensity at ground level. The third problem is related to an essential problematics in wing design : simultaneous drag and structural weight reduction. In all three cases, the meta-model-assisted MGDA succeeds in a few updates of the meta-model database to provide a correct description of the Pareto front, thus in a very economical way compared to a standard evolutionary algorithm used for this purpose.

Keywords: Differentiable optimization, numerical methods, multi-objective optimum-shape design, Pareto optimality, compressible aerodynamics, drag reduction, sonic boom 


\section{Introduction}

In multi-objective optimization [31], the notion of dominance in efficiency is classically introduced to define a partial hierarchy between solutions in the design space. Considering the simultaneous minimization of the objective functions $\left\{J_{i}\right)(i=1, \ldots, n)$, the design-point $Y^{(1)}$ is said to dominate in efficiency the design-point $Y^{(2)}$, iff

$$
J_{i}\left(Y^{(1)}\right) \leq J_{i}\left(Y^{(2)}\right) \quad(\forall i)
$$

and at least one of these inequalities holds strictly. In a non trivial multiobjective problem, no design-point exists that dominates all others, but design-points that are dominated by no other one do exist, and constitute the so-called Pareto front. The Pareto front is an extremely valuable information for the design engineer and devising efficient methods to identify it offers the numerical analyst an important methodological challenge.

In numerical optimization, two main families of approaches are commonly employed, both bearing advantages and disadvantages of their own : gradient-based methods and evolutionary strategies (see e.g. [1]).

In the literature, gradient-based methods have appeared rather early. They are very efficient tools for local optimization. When the initial guess is sufficiently close to the optimum solution, and the gradient available, they usually result in fast iterations to achieve an accurate convergence to the desired optimum. However these methods are usually not very robust, since they rely on the local convexity of the problem. Additionally in PDEconstrained optimization, developing the exact or approximate expression for the functional gradient is always a complex task. This difficulty is even more 
severe in multi-objective problems when the convexity or even the continuity of the Pareto front is not guaranteed.

Evolutionary strategies (ES) are semi-stochastic iterations relying on function evaluations only. In their principle, operators that imitate evolution (e.g. natural selection and mutation) are constructed to let a population of designpoints evolve according to successive generations. In general, these methods are less accurate, very demanding in computational effort, but greatly superior in robustness. An additional advantage of ES is to offer an easier framework for their extension, or transposition to the multi-objective context, because they solely rely on function values as does the above notion of dominance in efficiency. In [3], the best-known ES algorithms are compared on a few representative multi-objective test cases (PESA, SPEA2 [4], NSGA2 $[5])$.

The present article follows [8] [11] [12] in which a novel numerical method for multi-objective differentiable optimization, the Multiple-Gradient Descent Algorithm (MGDA), has been proposed to identify Pareto fronts. In MGDA, a direction of search for which the directional gradients of the objective functions are all negative, and often equal by construction [12], is identified and used in a steepest-descent-type iteration. The method converges to Pareto-optimal points [8]. These theoretical properties are briefly reviewed and applied first to a classical mathematical test-case for illustration.

In PDE-constrained optimization, as in particular, in optimum-shape design in aerodynamics, calculating the functional gradient by chain's rule or an adjoint-equation approach, is a computational endeavour, and a shortcut 
exists consisting in the construction of a local meta-model for the objective function yielding an approximate gradient. In this article, we investigate this option in the context of application of MGDA to multi-objective optimization problems.

Thus after demonstrating MGDA, we develop a strategy to couple MGDA with adaptively updated Kriging meta-models to compute approximate gradients. Kriging models are very commonly used in aerodynamic optimization. The meta-model assisted MGDA is successfully tested on a classical two-criterion, two-point aerodynamic optimum-shape design of lift and drag optimization. We then focus on two particular optimum-shape design problems : (i) the shape optimization of a supersonic wing body configuration to reduce the sonic boom intensity along with the aerodynamic drag; (ii) the aero-structural optimization of a wing body configuration to reduce the structural weight and the drag. 


\section{Multiple-Gradient Descent Algorithm (MGDA)}

\subsection{MGDA principle}

We consider the problem of simultaneous minimization of $n$ objective functions of $N$ design variables, $J_{i}(Y)\left(i=1, \ldots, n ; Y \in \mathbb{R}^{N}\right.$, design vector $)$. The dimensions $n$ and $N$ are arbitrary, although in many applications $n \leq$ $N$. Let $Y^{0}$ be a particular design-point about which the objective functions are smooth (say $C^{2}$ in practice) and locally convex. Denote $u_{i}^{0}=\nabla J_{i}\left(Y^{0}\right)$ $(i=1, \ldots, n)$ the gradients, and define the following convex hull:

$$
\overline{\mathrm{U}}=\left\{w \in \mathbb{R}^{N}, \quad w=\sum_{i=1}^{n} \alpha_{i} u_{i}, \quad \sum_{i=1}^{n} \alpha_{i}=1\right\} .
$$

$\overline{\mathrm{U}}$ is a closed, bounded and convex set associated in the affine space $\mathbb{R}^{N}$ with a polyhedron of at most $n$ vertices. Hence $\bar{U}$ admits a unique element of minimum norm, say $\omega[8]$. Two cases are possible:

1. $\omega=0$, and we say that $Y^{0}$ is a point of Pareto-stationarity a necessary condition for Pareto-optimality;

2. or $\omega \neq 0$, and the directional derivatives of the objective functions satisfy the inequalities:

$$
\left(u_{i}^{0}, \omega\right) \geq\|\omega\|^{2}
$$

hence, $-\omega$ is a descent direction common to all the objective functions.

In the latter case, we define MGDA as the iteration that uses $-\omega$ as the direction of search, and a step-size adjusted to maximize the smallest absolute decrease of the criteria. Accumulation points of this method are 
Pareto-optimal designs [8] [11]. In this way, MGDA generalizes to the multiobjective optimization the classical steepest-descent method [1].

In the particular case of two criteria, the minimum-norm vector is known analytically. Figure 1 then shows vector $\omega$ in the three different possible cases.
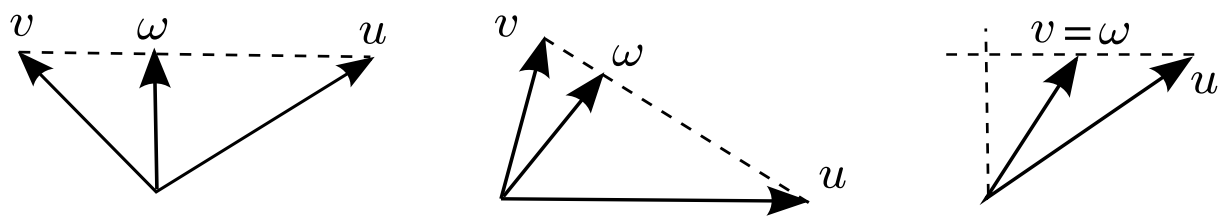

Figure 1: Various possible configurations of the two gradients-vectors $u=u_{1}$ and $v=u_{2}$ and the minimal-norm element $\omega$.

As a first illustration of the method, several analytical multi-objective optimization test-cases proposed in [3] have been solved by MGDA [9], and some of these results are presented next in comparison with an evolutionary strategy.

\subsection{Analytical validation}

The test-case corresponds to the two-objective unconstrained minimization of the following functions :

$$
\left\{\begin{array}{l}
f_{1}(Y)=1-\exp \left(-\sum_{i=1}^{3}\left(y_{i}-\frac{1}{\sqrt{3}}\right)^{2}\right) \\
f_{2}(Y)=1-\exp \left(-\sum_{i=1}^{3}\left(y_{i}+\frac{1}{\sqrt{3}}\right)^{2}\right)
\end{array}, \quad Y=\left(y_{1}, y_{2}, y_{3}\right) .\right.
$$

The design variable is $Y=\left(y_{1}, y_{2}, y_{3}\right) \in \mathbb{R}^{3}$. This test-case is known to yield a continuous but non convex Pareto set in function space. The Pareto front was identified by Deb using the well-known genetic algorithm NSGA-II [3]. 

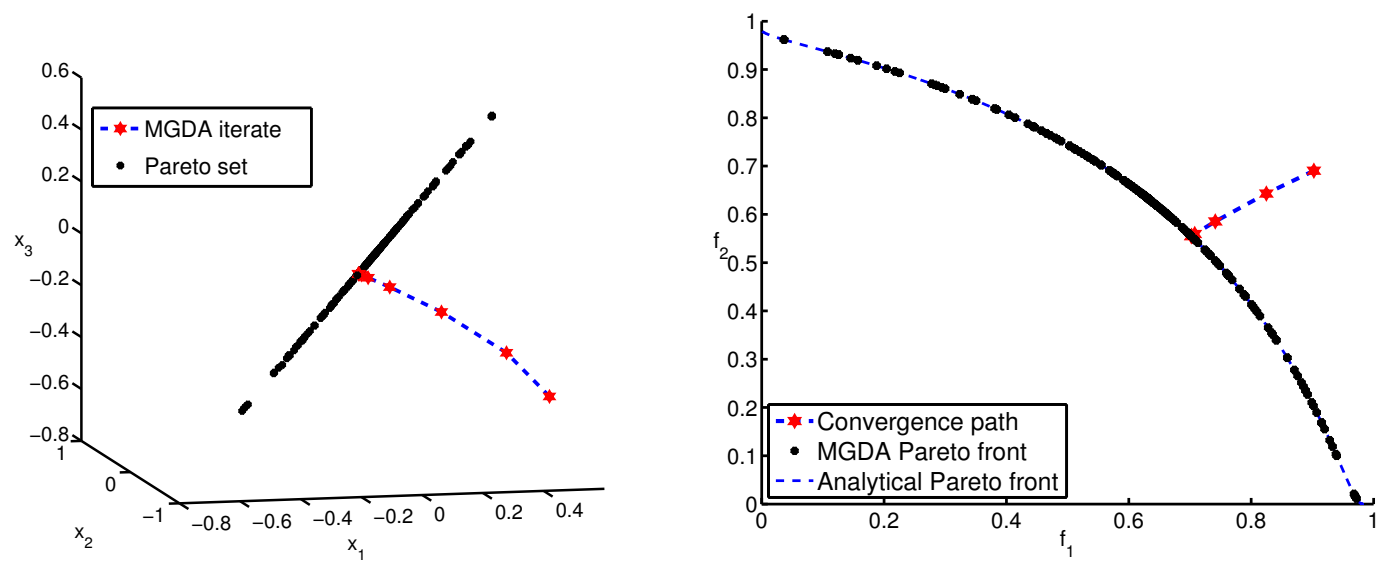

Figure 2: Convergence of MGDA from an initial design point to the non dominated set.

From a given starting point, MGDA converges quickly (6 steps in this example) and provides an accurately-defined design-point on the Pareto set.

After applying the method from a set of 60 initial design-points distributed over a sphere in the design space (see Figure 3), we have obtained an accurate discretization of the known-analytically Pareto set.

\subsection{Meta-model-assisted MGDA}

In PDE-constrained optimization, and in particular in optimum-shape design in aerodynamics, the calculation of function values and their gradients can be very computationally demanding, and usually requires substantial methodological developments. To alleviate this task, in this article, we investigate the possibility of calculating approximate gradients from a surrogate model, or meta-model, devised from a database of high-fidelity function values. In the applications considered presently, the high-fidelity models are associated with 3D compressible flows governed by the Euler or RANS equations. 

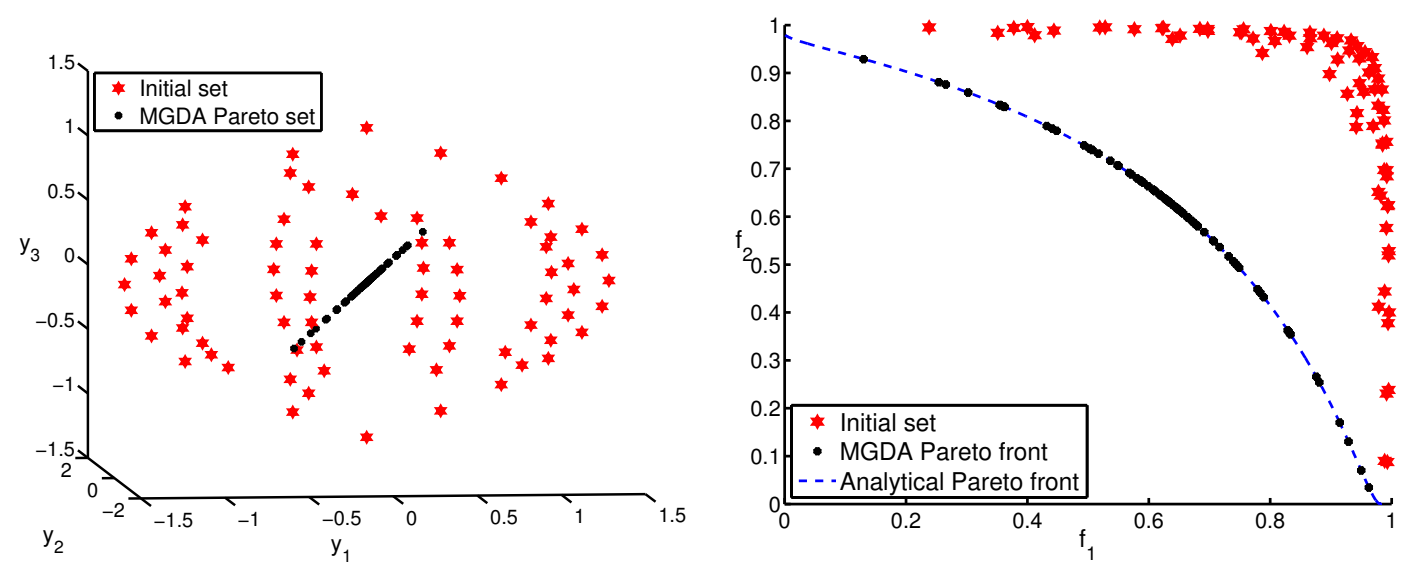

Figure 3: Convergence of MGDA from initial design points around Pareto front, for a classical test case proposed by Fonseca, in design space (left), in function space (right).

We proceed as follows (see flowchart in Figure 4). An initial set of design points is generated using of a latin hypercube sampling in $\mathbb{R}^{N}$. The sampling serves two purposes. Firstly, the function values corresponding to the sampling form a database supporting Kriging meta-models, surrogate of the actual objective functions. Secondly, some of these sampling points are used to initiate independent MGDA iterations applied to the multi-objective minimization of the meta-models, and converging to Pareto-stationary points (associated with the meta-models). These Pareto-stationary points are then evaluated according to the high-fidelity models to enrich the database and proceed with the next update. A filtering method is used additionally to remove points found too close to an existing design-point, in order to avoid redundancy. 


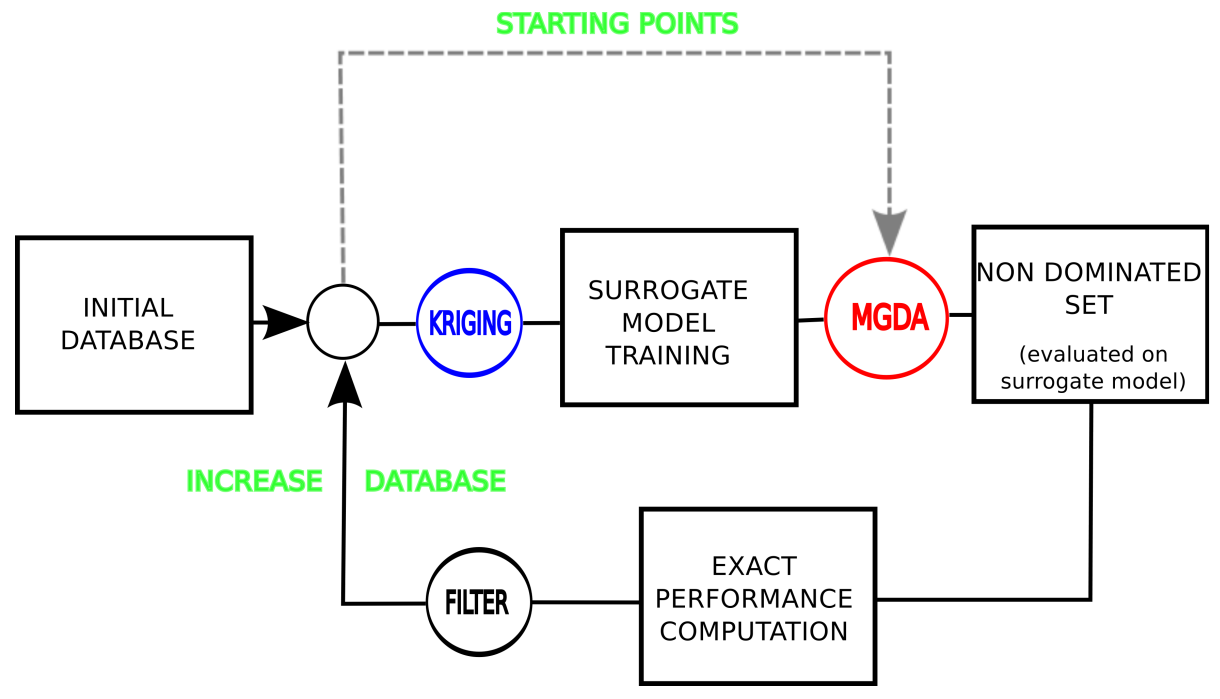

Figure 4: MGDA with surrogate model scheme. A surrogate model based on an initial database is trained. Then, MGDA [8] is applied from each database point. Thus a non dominated set on the surrogate model is obtained.

\subsection{Application to optimum-shape design for lift and drag optimization}

To illustrate the meta-model-assisted MGDA, we consider a two-objective and two-point shape optimization of a generic aircraft wing. The flow field is governed by the 3D compressible Euler equations solved by finite volumes over an unstructured mesh. The optimization problem consists in maximizing the lift coefficient in subsonic conditions $\left(M_{\infty}=0.2, A o A=8^{\circ}\right)$ together with minimizing the drag coefficient in transonic conditions $\left(M_{\infty}=0.8\right.$, AoA $=2^{\circ}$ ) subject to bound constraints on the geometrical variables.

The three-dimensional geometry is generated from an airfoil, the wing cross sections being made two by two homothetic assuming a linear variation in the span-wise direction. Thus only the shape in a given section is subject to the optimization. The reference shape is a NACA0012 airfoil approximated 
via $5 \mathrm{~B}$-spline functions for the upper surface, and 5 other ones for the lower surface, see (figure 5). The same 10 locations are associated with 10 degrees of freedom, permitting constrained vertical displacements. The leading and trailing edges are maintained fixed.

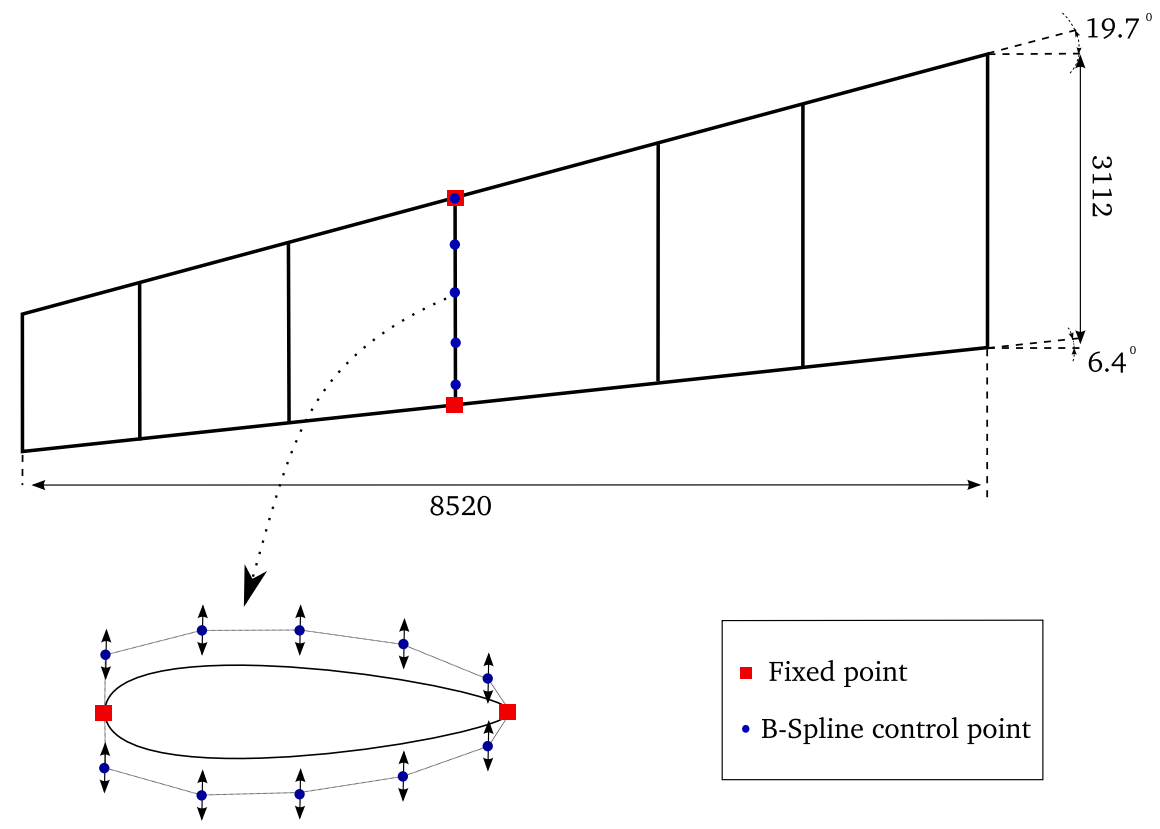

Figure 5: B-Spline shape parametrization through a few control points.

In this numerical experiment, a database of 40 design-points in $\mathbb{R}^{10}$ has been used to initiate the process. Kriging meta-models are constructed for lift in the subsonic conditions and drag in the transonic conditions. Figure 6 shows the step-by-step meta-model assisted MGDA convergence with exact performance re-evaluation. The non dominated set is indicated for the initial and final databases for comparison, indicating a progressive trend towards the Pareto front. In this experiment, a total 223 geometries have been evaluated via 446 flow-field computations. Among the final non-dominated solutions, 
the achieved least drag coefficient was equal to $2.13 \times 10^{-3}$, and the maximum lift to 0.836 .

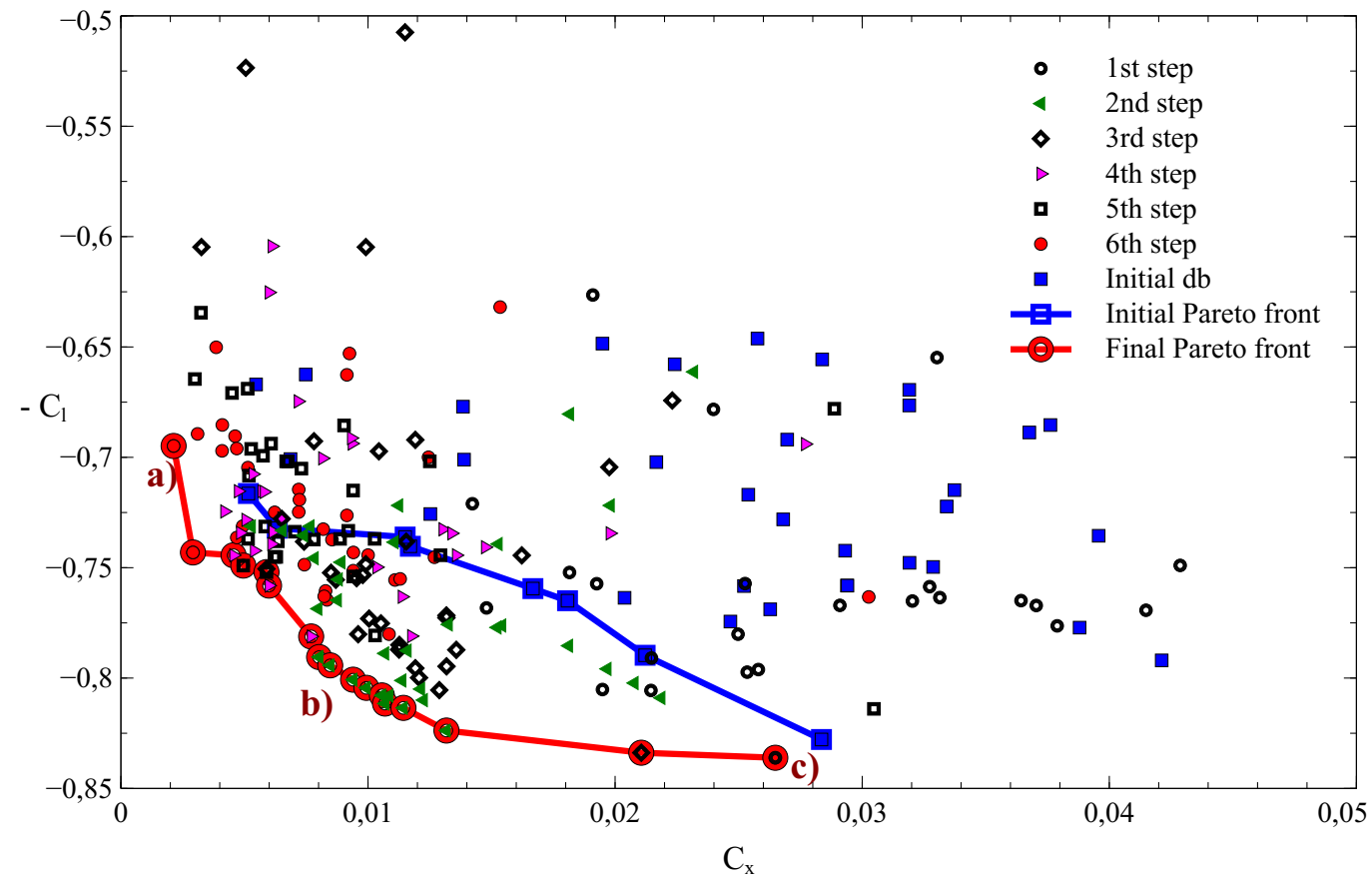

Figure 6: MGDA on surrogate model evolution on a classical drag coefficient minimization and a lift coefficient maximization. Comparison between the initial and final non dominated sets.

Figures 7 and 8 provide the pressure distribution in the transonic and subsonic conditions respectively. Three different span-wise positions , 5\%, $50 \%$ and $95 \%$ are illustrated and compared with the pressure distribution corresponding to the initial geometry.

Unsurprisingly, the minimum-drag configuration is associated with the weakest shock-wave, and the maximum-lift configuration with the thickest airfoils. 
a) Lowest drag
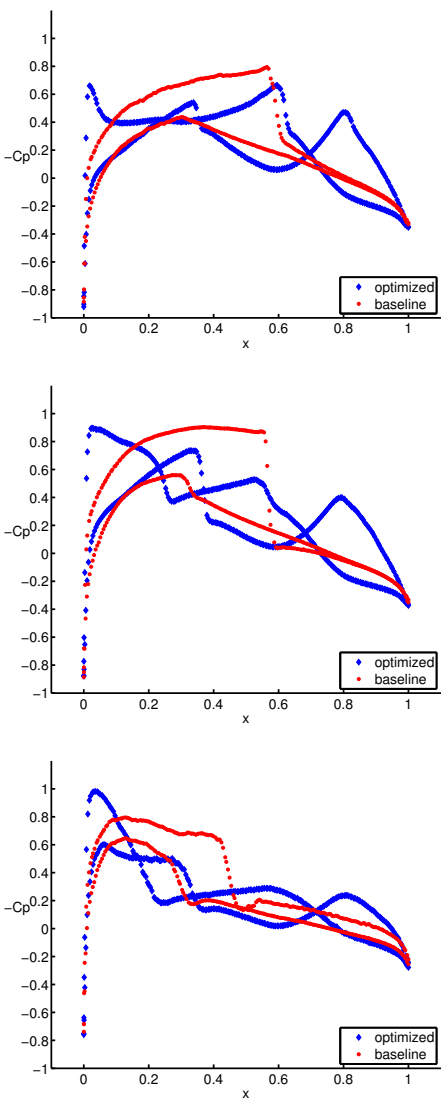

b) Intermediate
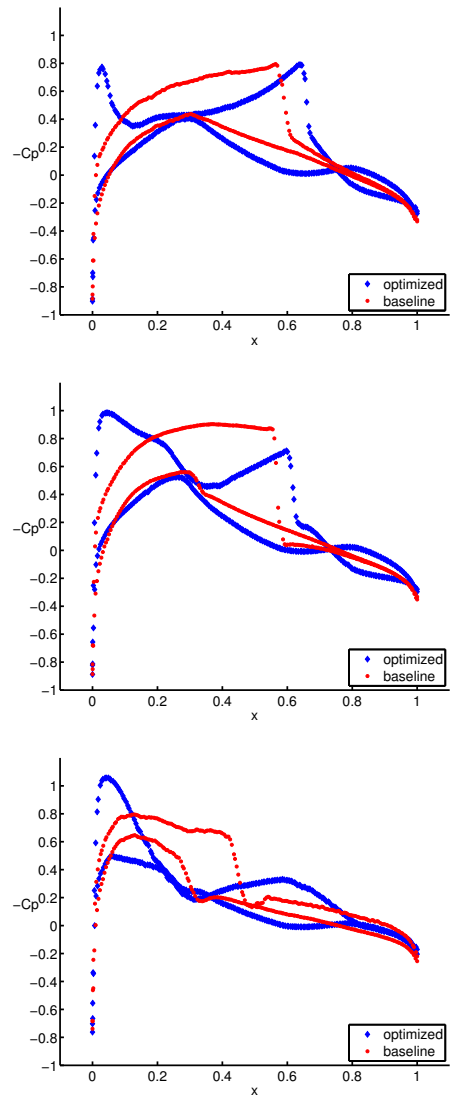

c) Highest lift
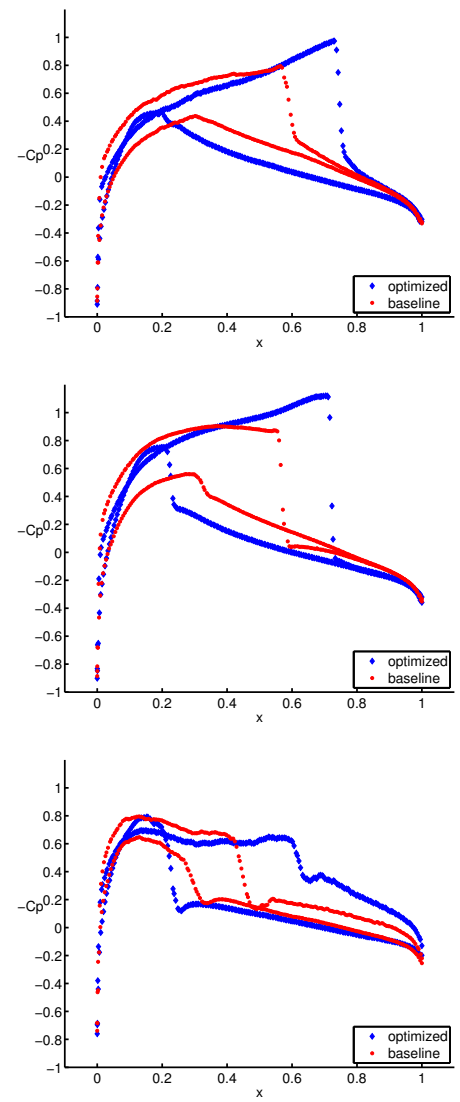

Figure 7: Pressure coefficient distributions evaluated in the transonic conditions $\left(M_{\infty}=\right.$ 0.83, $A o A=2^{\circ}$ ) and corresponding to the minimum-drag configuration (left), maximumlift configuration (right) and an intermediate configuration (middle). Top/middle/bottom : $5 \% / 50 \% / 95 \%$ spanwise.

In conclusion, this preliminary experiment has demonstrated the efficacy of the meta-model-assisted MGDA to approximate the Pareto front from an initial dataset of design points in a few database enrichment cycles. 
a) Lowest drag
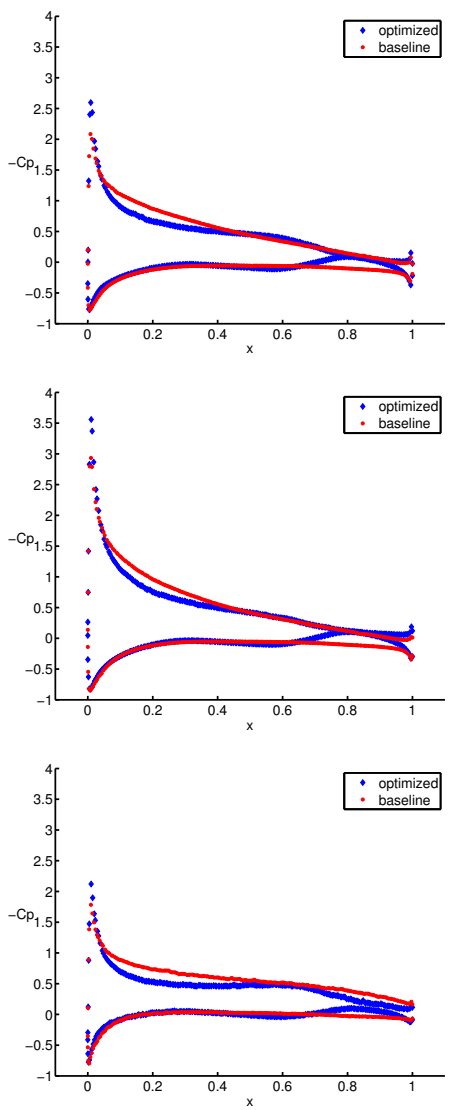

b) Intermediate
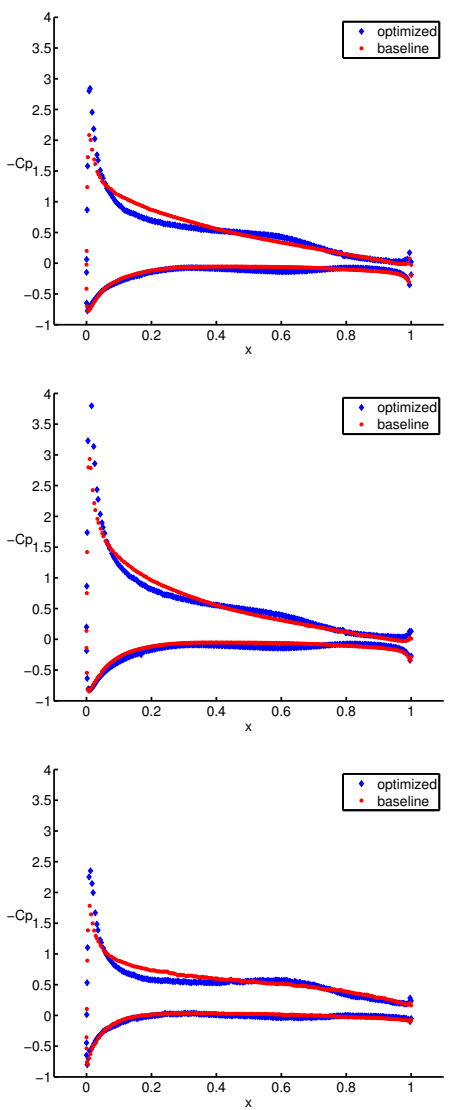

c) Highest lift
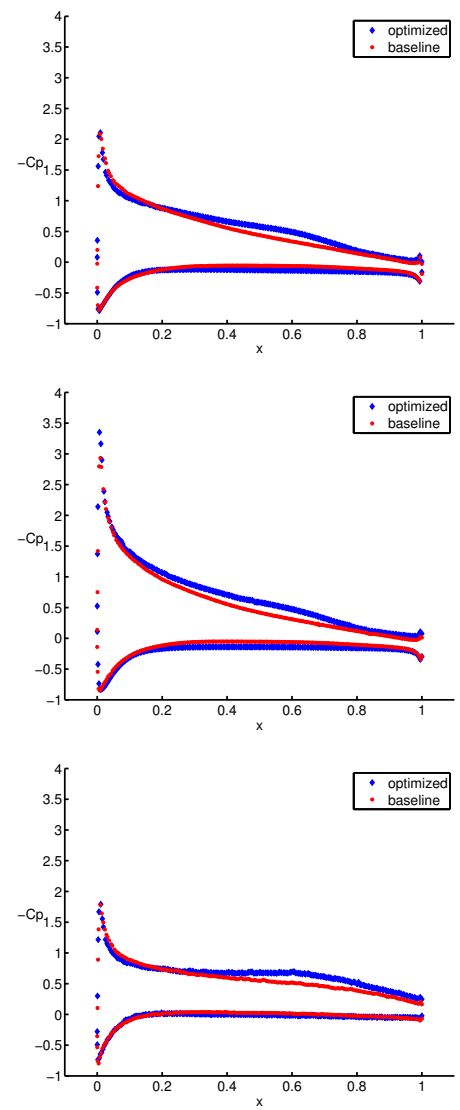

Figure 8: Pressure coefficient distributions evaluated in the subsonic conditions $\left(M_{\infty}=\right.$ 0.30, $A o A=8^{\circ}$ ) and corresponding to the minimum-drag configuration (left), maximumlift configuration (right) and an intermediate configuration (middle). Top/middle/bottom : $5 \% / 50 \% / 95 \%$ spanwise. 


\section{Application to industrial problems}

MGDA has been introduced and validated on analytic functions and on a simple shape design test case. Due to its efficiency in terms of limited number of function evaluations required for obtaining a description of the Pareto front, it is well suited for real life industrial problems based on high fidelity methods. In the following sections two different applications to multiobjective shape optimization problems are presented.

\subsection{Aeroacoustic shape optimization}

The development of civil supersonic transportation implies today several technological challenges, but one of the most limiting factor is the environmental impact in terms of sonic boom [13] [14].

Since March 1973 supersonic flight overland by civil aircraft has been prohibited in the United States (FAR 91.817). Enough progress on reducing impact of sonic boom before reach the ground have been achieved during the years, but the problem still persists. The problem of sonic boom minimization has been investigated since the fifties and still, nowadays, there is no common practice to analyze and optimize the shape in order to reduce this deleterious phenomenon.

Considering an aircraft travelling at a speed superior to the local speed of sound and a reference system in motion with the aircraft, the body produces a pressure perturbation in a conical region, called Mach cone that starts at the nose of the aircraft and intersects the ground forming the primary carpet. This is the region on ground that have the strongest level of perceived loudness and it has an extension from 10 to $100 \mathrm{~km}$, as a function of 
the aircraft configurations, flight and atmospherical conditions. Looking at the signal just below and near the aircraft each geometry discontinuity such as wing, engine nacelle and control surfaces is responsible of multiple complex shocks and expansion waves patterns. During the propagation of these

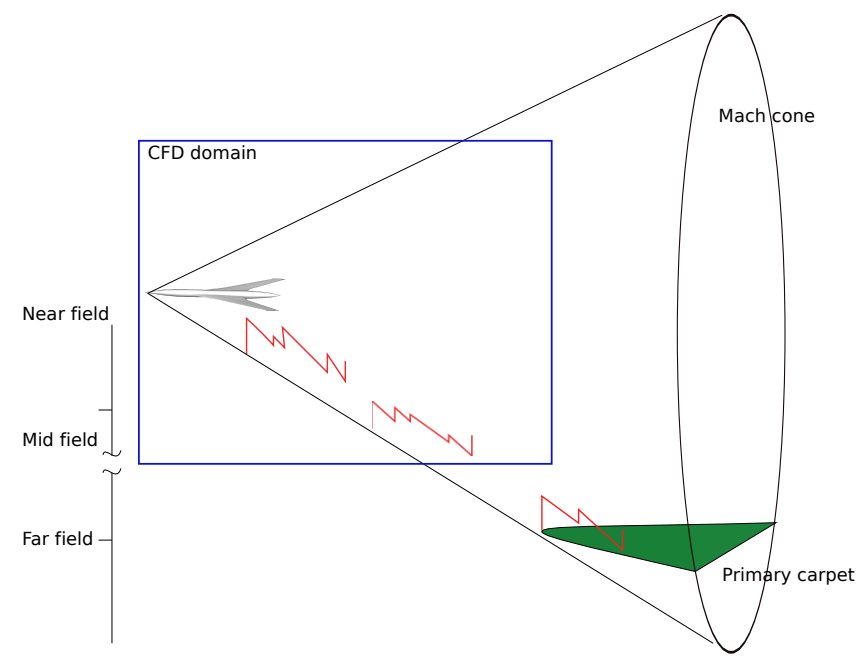

Figure 9: Mach cone and CFD domain.

pressure disturbances to the ground through a non uniform atmosphere, different dominating physical phenomena act to modify the acoustic signature. Three different regions can be identified: the near-field, the mid-field and the far-field (see figure 9). The near-field is the region close to the aircraft whitin some body lengths where the non linear inviscid three dimensional aerodynamic perturbation theory applies far from the boundary layer region, generally described using Navier-Stokes equations. The mid-field is the region where the non linear behaviour of the compressions and expansions are not negligible. This domain where the flow can be considered inviscid 
extends from few to hundreds body lenghts. The signal propagates through this region and reach the ground in the far-field. The typical signature undertrack follows an N-wave pattern, which consists in a shock followed by a linear expansion and a subsequent pressure rise which restores ambient pressure. Atmospheric non uniformity, turbulence and aircraft maneuvers produce drastic variations of the waveform [15]. In the present work only steady level flight in standard stratified atmosphere conditions without wind is considered.

One of the most challenging aspect in the modelisation of this phenomenon is related to the different scales between aerodynamics and acoustic disturbances coupled with a problem domain of more than $10 \mathrm{~km}$. Despite the extension of the domain and the difficulties highlited some experiences have been performed. Cheung et al. [16] already in the nineties proposed the use of parabolized Navier-Stokes equations to predict the mid flow-field pointing out the expensive computational cost. CFD computations using Euler and full-potential equations to the far field evaluation and mesh adaptation have been evaluated on simple configurations by Kandil et al. [17]. Despite the continuous increase in computational power, a brute force complete CFD evaluation for real aircraft geometry is still challenging and appears useless if no counter-measures based on mesh adaptation are taken into account.

The most accurate and efficient method available today is based on three layer decomposition methods [18]. It is based on the coupling of an aerodynamic and acoustic model. The 3D CFD computation is performed in the near-field. The sonic boom signal is propagated down to the ground after a near to far-field matching method that makes the pressure near-field 
compliant with the acoustic model adopted for the propagation. The farfield propagation is performed using non linear acoustic and in particular the ray-tracing technique [19]. In the following analysis the near-field domain is evaluated solving Euler equations with elsA [20], the ONERA in house CFD solver. The pressure near field is then interpolated on a cylinder that surrounds the aircraft and used as input for the acoustic code TRAPS [19]. This code performs the propagation through the stratified atmosphere.

In optimization problems this technique is computationally expensive in particular in preliminary design phases, because of the high number of function evaluations required. For this reason an efficient optimization algorithm is required.

\subsubsection{Supersonic glider "low boom - low drag" shape design}

The application case consists in a wing body configuration with the following flight conditions: Mach number equal to $M_{\infty}=1.6$, flight altitude of 18,000 $\mathrm{m}$ and an AoA equal to 2 degrees. The reference geometry considered shows an overall length is $30 \mathrm{~m}$ with a wingspan of $18 \mathrm{~m}$. The fuselage is axi-symmetric with cylindrical cabin of length and radius respectively equal to $11 \mathrm{~m}$ and $2.03 \mathrm{~m}$. The fuselage geometry is obtained using a control line that is revolved along the longitudinal axis. The control line is defined using three tension splines curves in order to describe the nose, the middle of the cabin and the rear part of the fuselage. The wing is a double swept wing that shows a diedral angle of the outer wing of 3 degree. The wing has a double convex profile with $\alpha_{0}$ equal to zero. Along the wingspan no twist is applied, while the thickness-to-chord ratio $t / c$ evolves linearly from 0.6 to 0.3 . Figure 10 shows the set of design variables adopted to describe the 


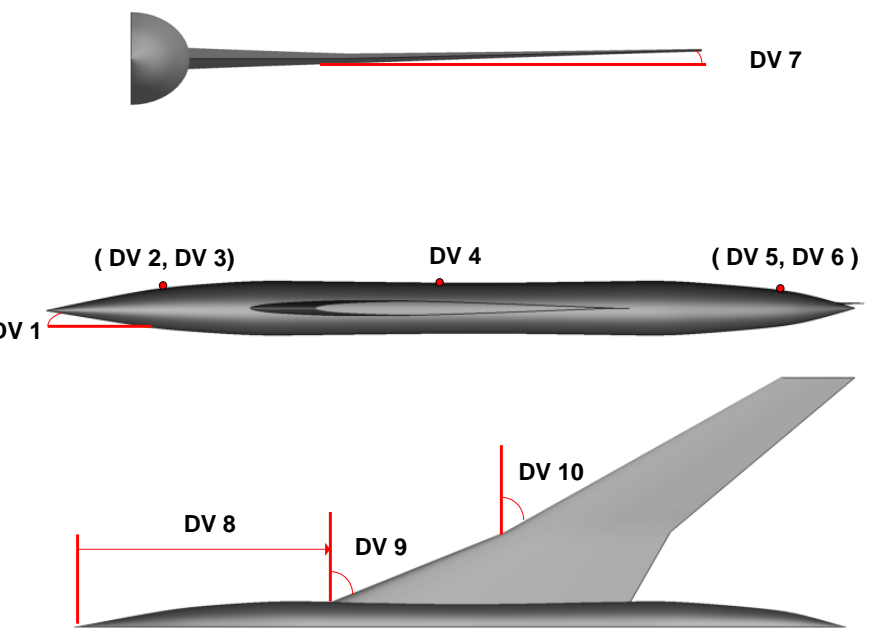

Figure 10: Supersonic glider parameterisation

complete geometry. The first six design variables (DVs) define the fuselage and consists in the nose deflection angle $\alpha_{n}$, a control point on the nose, one in the middle that has a fixed $\mathrm{x}$-coordinate and another control point that describes the rear of the fuselage. The wing planform geometry is defined using the leading edge sweep angle of the inboard and the outboard wing respectively $\phi_{B A}^{1}$ and $\phi_{B A}^{2}$, the longitudinal position of the wing $x_{w}$ and the dihedral angle $\Lambda$ of the external wing. The set of design variables is written as :

$$
D V=\left(\alpha_{n}, x_{1}, z_{1}, x_{2}, x_{3}, z_{3}, \Lambda, x_{w}, \phi_{B A}^{1}, \phi_{B A}^{2}\right)
$$

The reduced number of variables is an implicit requirements to the construction of a surrogate model with a limited number of individuals in the starting database. The initial database consists of 50 individuals and it has been created using a classical LHS method. The DV set represents different 
geometrical entities: angles, relative distances and length. Instead of their direct use in the optimization problem, to provide a better conditioning of the problem, a non-dimesional optimization variables set $\mathbf{x}$ is adopted. The optimization variables $\mathbf{x}$ are related to the geometry DVs using the following equation:

$$
D V_{i}=\bar{D} V_{i}\left(1+x_{i} \delta_{i}\right) \quad \forall i>1
$$

where $\overline{D V}{ }_{i}$ represents the geometrical variable of the reference configuration, $\delta_{i}$ is the maximum percentage modification allowed to each variable. An exception is made for the nose deflection variable because $\overline{D V_{1}}$ is equal to 0 , the following relation is adopted: $D V_{1}=0.5 x_{1}$. The design variables set $\mathbf{x}$ can assume values between -1 and 1 . Table 1 shows the geometrical variables and their respective modification allowed.

\begin{tabular}{|cccc|}
\hline \hline Variable & Specification & $\overline{D V_{i}}$ & $\delta_{i}$ \\
\hline DV1 & nose deflection [deg] & 0 & - \\
DV2 & x-coordinate nose section [m] & 4 & $2 \%$ \\
DV3 & radius nose section [m] & 0.8 & $10 \%$ \\
DV4 & radius cabin section [m] & 1.015 & $10 \%$ \\
DV5 & x-coordinate rear section [m] & 28 & $2 \%$ \\
DV6 & radius rear section [m] & 0.535 & $10 \%$ \\
DV7 & dihedral angle [deg] & 3 & $100 \%$ \\
DV8 & relative wing position [adim] & 0.367 & $10 \%$ \\
DV9 & inner wing sweep angle [deg] & 65 & $10 \%$ \\
DV10 & outer wing sweep angle [deg] & 56 & $10 \%$ \\
\hline \hline
\end{tabular}

Table 1: Design variables set definition. 
A common and accepted metrics that is able to describe the outdoor impact of sonic boom is not established today. Pressure based and loudness metrics such as A-SEL or PLdB are the most common [22]. The sum of the pressure shocks amplitude is the metric adopted due to its direct relationship with the ground signature shape, the simplicity and the ability of the parameterization to describe the entire geometry and as a consequence shape the complete undertrack ground signature. A similar metric based on the maximum shock amplitude of the ground signature has been proposed for the European project HISAC [23]. The typical front shock pressure objective is a subset of this general case and the corresponsing solutions belongs to the final set at the convergence of the optimization algorithm to the Pareto set. The objective function for aerodynamics and acoustics are respectively the drag coefficient and the sum of the shock overpressures of the undertrack ground signature. A lift constraint is also considered. The problem can be stated as follows:

Minimize $\left\{\begin{array}{lll}J_{1}(\mathbf{x})=c_{D} & \text { subject to : } g: c_{L}-c_{L_{0}} \geq 0 \quad \text { with } c_{L_{0}}=0.1 \\ J_{2}(\mathbf{x})=\sum \Delta p & \text { subject to : } & \text { no constraints }\end{array}\right.$

The same strategy described in the first part is applied to solve the aeroacoustic optimization. At each step the algorithm tends to converge toward the optimal front of the two objectives optimization problem based on the surrogate models. This set of solutions are then evaluated using the high fidelity CFD-based three-layer approach. These new points in addition with the database at the $i^{\text {th }}$ iteration are then used to create a new surrogate model at the iteration $i+1$. In this application all the training points of the 
low fidelity model are used as MGDA starting point. This aims to increase the size of the non dominant points which are identified, thus reducing the number of MGDA steps required. The algorithm does not take into account bound contraints on the variable ranges. A filter on the feasible solutions that respect the variables range at the end of each step on the surrogate model is required. Furthermore, an adequate initial step choice of the descent iteration prevent the convergence outside the feasible domain. Figure 11 shows the convergence of MGDA toward the Pareto front . After the first step of MGDA, each new set brings improvement in the Pareto optimal thus increasing the number of non-dominated points. The first step is applied on a surrogate model made only by 50 points, this could explain the sparsity of this step. Six step of the metamodel assisted MGA are sufficient for the

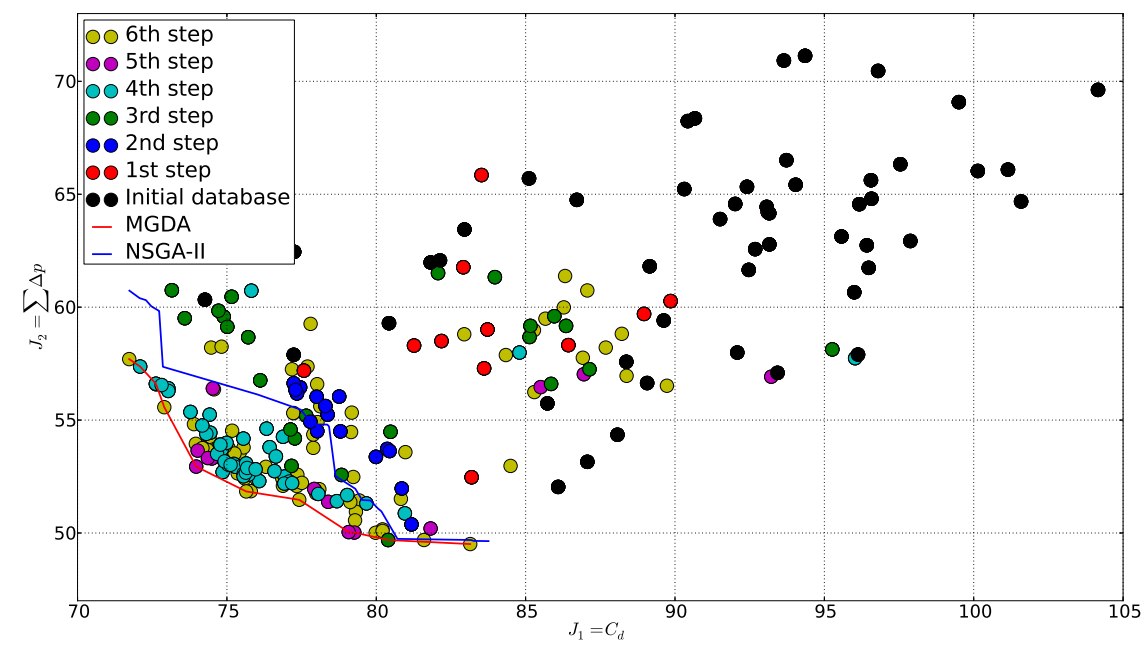

Figure 11: Convergence history at fixed maximum high fidelity function evaluation calls.

clear definition of the Pareto front. 
The NSGA-II algorithm applied on the high fidelity model is used as comparison in order to analyze and compare the solution evaluated using MGDA. The number of evaluations on the high fidelity model used for MGDA convergence is used as stop criterium for NSGA-II. This means that the comparison is made at identical computational cost. Figure 11 clearly shows that the Pareto front evaluated using MGDA completely dominates the solution of NSGA-II, thus identifying the efficiency of MGDA. The metamodel assisted MGDA requires less function evaluations during the convergence to the real Pareto front and is suited to computationally expensive problems. Furthermore, the diversity of the solutions is comparable between the two algorithms.

The convergence of MGDA on the low-fidelity model is shown in figure 12. Four points are used as starting points to show the limited amount of function evaluations required by this gradient based algorithm to converge to the Pareto front. In addition, the mixed-fidelity approach based on the continuous update of the surrogate model assures an accurate description of the real model, despite the well known difficulties encountered with a noisy, non smooth, with multiple local minima cost function such as the sonic boom one. Figure 13 shows the comparison between the points evaluated using the surrogate model at the last step of the algorithm and the corresponding set obtained after the high-fidelity model evaluations.

Three different configurations (see table 2) that belong to the Pareto front are compared in figure 14 and 15. The low drag configuration (A) and the trade-off configuration (B) show minor modifications on the fuselage geometry. The modification impacts mainly the wing planform. Configuration (B) 


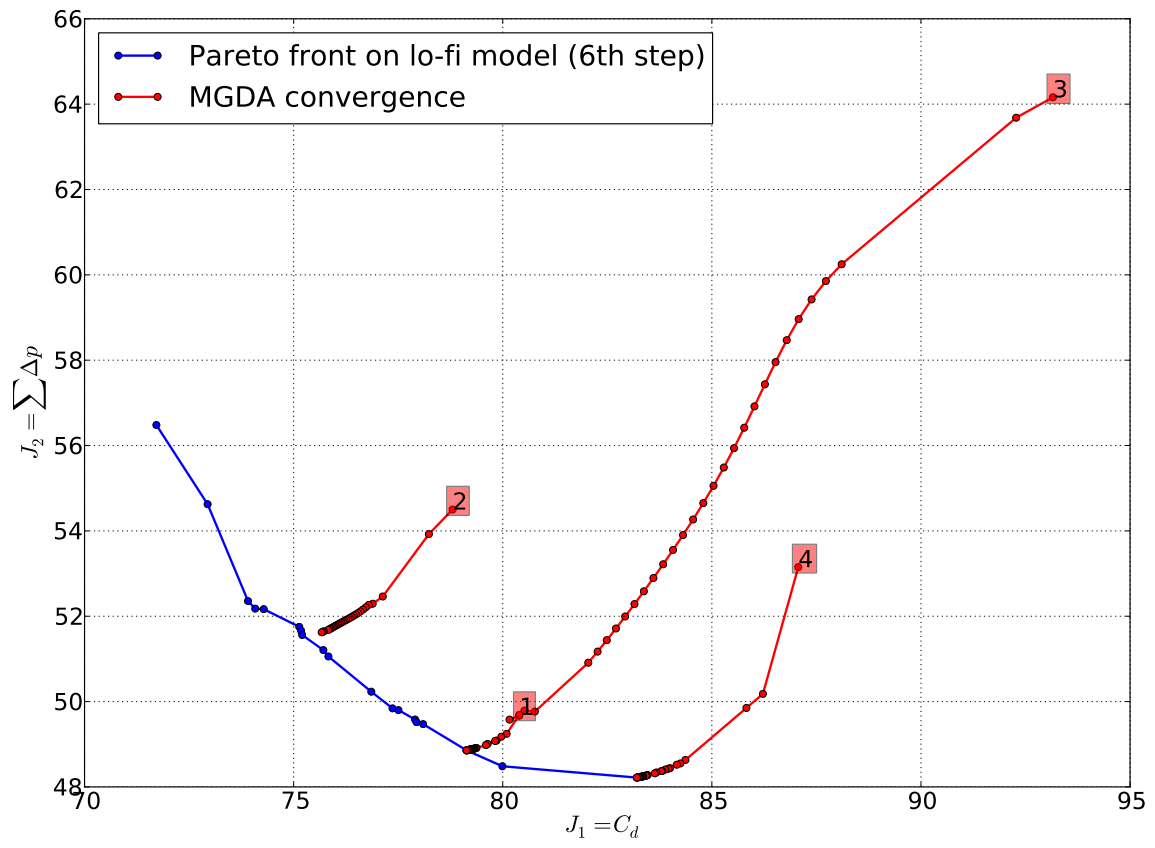

Figure 12: MGDA convergence to the Pareto front of the low-fidelity model starting from 4 initial point of the database.

\begin{tabular}{|ccc|}
\hline \hline Configuration & $J_{1}$ & $J_{2}$ \\
\hline A & $71.72 \mathrm{dc}$ & $57.7 \mathrm{~Pa}$ \\
B & $73.98 \mathrm{dc}$ & $52.94 \mathrm{~Pa}$ \\
C & $83.14 \mathrm{dc}$ & $49.51 \mathrm{~Pa}$ \\
\hline \hline
\end{tabular}

Table 2: Selected configurations that belong to the Pareto front.

shows a reduction of the dihedral angle, with respect to the configuration (A) that is benefical for the rear shock of the ground signature (see figure 15(b)) with a limited deterioration on the aerodynamic performance. Indeed, the dihedral angle acts modifying the acoustic footprint at ground, in particu- 


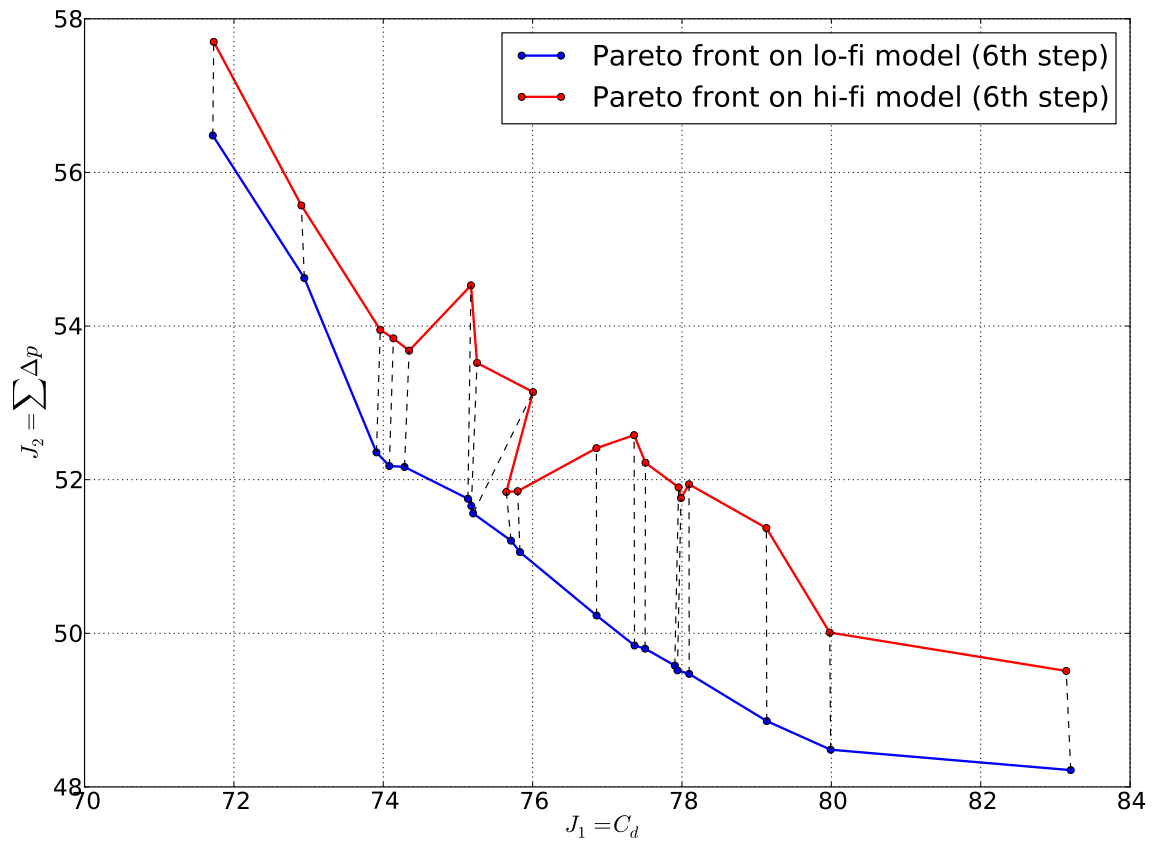

Figure 13: Comparison between the Pareto front of the MGDA 6th step and the values after high-fidelity evaluations

lar the duration of the signature. The low boom configuration $(\mathrm{C})$ shows a strong downward displacement of the nose angle, which has as a consequence a modification of the flow condition that reaches the wing leading edge. The front shock is almost not modified by any of the selected geometries. The algorithm has identified the wing and the rear shock reductions as the most promising regions to improve the functions of interest with respect to the chosen parameterisation As the sonic boom is reduced from (A) to (C), the expansion after the first shock is reduced. Looking at the pressure signal just below the aircraft in figure 15(a) it is possible to note that the design 


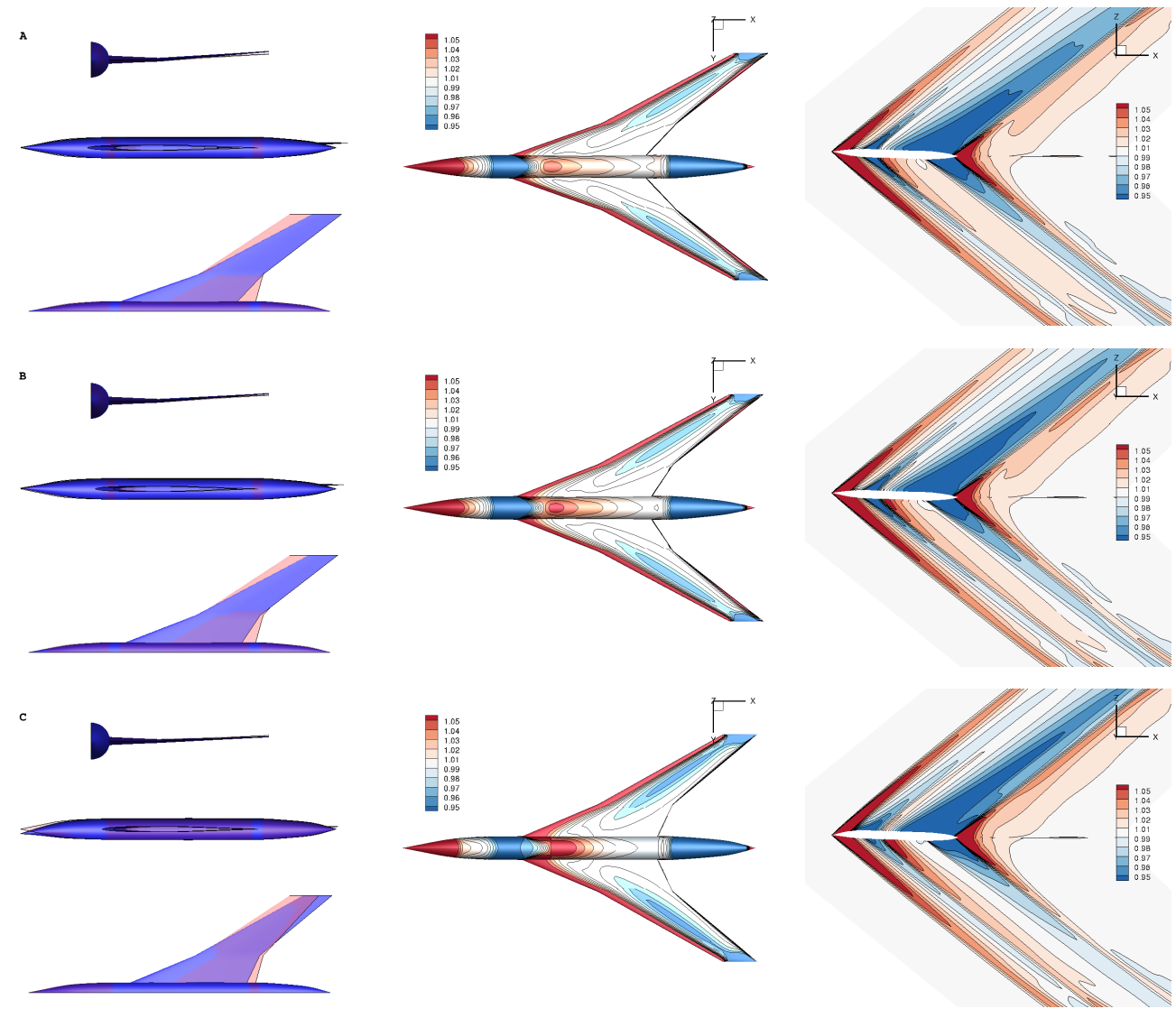

Figure 14: Geometry configuration, bottom skin pressure $\left(p / p_{0}\right)$ and symmetry plane pressure $\left(p / p_{0}\right)$ in near field for configuration $\mathrm{A}, \mathrm{B}$ and $\mathrm{C}$ on the Pareto front (In red the initial geometry).

variables are able to act and shape all the shock and expansion waves.

In particular all the configurations show a split of the rear shock due to the combined wing planform and rear fuselage modifications. The initial peak in the near field $p / p_{0}$ is reduced by the $20 \%$ with respect to the initial configuration, but this does not correspond to an analogue reduction of the front shock overpressure (figure 15(b)). In contrast, wing and bow fuselage mod- 


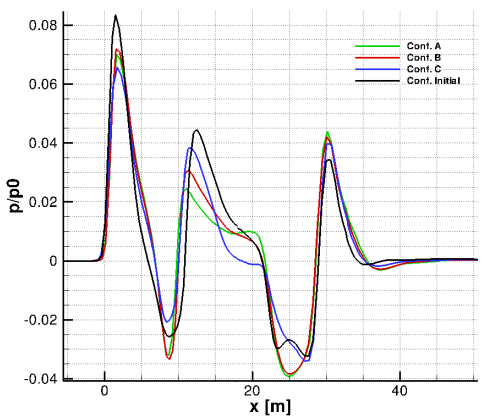

(a) Near field $p / p 0(\mathrm{z}=\mathrm{h}-10 \mathrm{~m})$

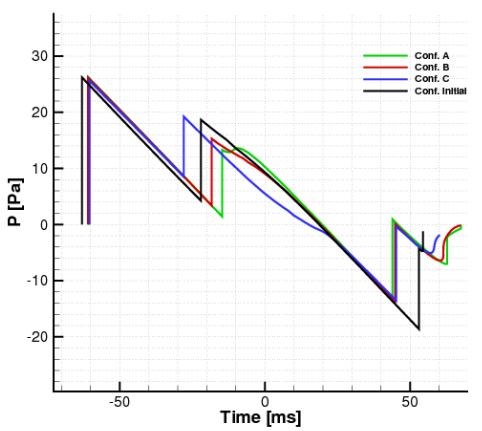

(b) Ground signature

Figure 15: Near-field and ground pressure signal for different configurations on the Pareto front

ification produces changes in the middle and ending part of the near-field signature, that correspond to strong modifications on the ground signature pattern. A better description of the nose shape with more design variables, can improve the ability of the algorithm to reduce the front shock amplitude.

The MGDA algorithm assisted with surrogate models to solve multiobjective problems where the objective functions of interest are expensive to evaluate and a limited number of calls to the high fidelity model has been applied to a complex supersonic non-viscous test case succesfully.

In the following section the aerodynamic model is improved considering Navier-Stokes equations in order to solve an aeroelastic viscous case for a civil transport wing-body configuration. 


\subsection{Aerostructural shape optimization}

An optimal wing will be extremely rigid to resists aeroelastic effects and considerably light for economical reasons. Unfortunately these two properties evolve monotonously in the same direction: the wings are flexible because of their high dimension (high aspect ratio), making them stiffer will come at the expense of structural weight. The task of the designer is then to find a balance in the design space, a wing light enough to meet the environmental and economical needs and rigid enough to meet the FAR25 safety standards. Wing structural and aerodynamic behavior are not only linked through performance. To keep the wing from excessive twist more structural material for strength and stiffness is requisit. The aerodynamic loads influence the structural wing deformations, and the wing planform influences both magnitude and distribution of aerodynamic loads. The direct conclusion is: when optimizing aircraft performance in terms of drag, the structure cannot be treated as an isolated system, it must interacts with aerodynamics. Because both weight and drag are unfavorable to flight performance, this work aims, through multidisciplinary optimization problem, to minimize both the near field drag and the structural weight under aerodynamic and structures constraints.

Drag versus structural weight minimization can be formalized as follows

$$
\begin{gathered}
\text { Minimize } C_{D}(\alpha) \quad \text { at } C_{l}=C_{l \text { Design }} \\
\alpha \in \mathbb{R}^{n} \\
\text { Minimize } W(\alpha) \quad \text { subject to } \\
\left\{\begin{array}{l}
\alpha \in \mathbb{R}^{n} \\
\sigma_{i}(\alpha) \leqslant \sigma_{\text {yield }}
\end{array}\right.
\end{gathered}
$$


Where $\sigma$ are the internal structural stresses and $\alpha$ the design variables that impact both objectives.

\subsubsection{Aerodynamic and structural design of the Airbus XRF1 configuration}

The selected 3D wing-body configuration is the so called XRF1 Airbus configuration. A structured mesh of 143 blocks is used (Fig 16(a)). A natural design space for aerostructural performance search is the conbination

of planform parameters $\alpha_{\text {geom }}$ (span, twist, sweep angle, etc...) and internal structure parameters $\alpha_{\text {struct }}$ (Skin thickness, front spar wel thickness, etc...) but a realistic design problem is also a highly constrained optimization problem, thus to restrict to the paper subject and provide a of MGDA efficiency, the wing of the XRF1 configuration is parameterised by wing span, sweep angle, and thickness to chord ratio at $y=8.399$ and $y=28.8$. The choice of this parametrisation, detailed in 3.2 .2 , is to impact significantly both objectives. In Euler computations the far field drag components are the induced drag $C D_{i}$ and the wave drag $C D_{w}$. The increase of the wing span comes with a decrease of $C D_{i}$ and an increase of the structural weight, regardless of wing area modification that is kept constant in this work.

An increase of the sweep angle reduces $C D_{w}$ by reducing the local mach number, the structural weight is increased by both the increase of structural span and the increase of the wing tip loading. A higher local thickness-tochord reduces the structural weight. In fact, to support the same bending loads the thicknesses of the elements sized in bending are decrased by the factor by which the wing thickness is increased. The local camber induced by a higher thickness to chord ratio increases the velocities and generates more $C D_{w}$. In conclusion all this parameters drive the objective functions 
in opposite directions and thus constitut an adapted case to test MGDA.

\subsubsection{Aerodynamic and structural analysis}

The aerodynamic analysis is perfomed by the structured code els $A$ [20] at Mach 0.83 targeting a lift coefficient of 0.5 . The target lift was selected to remove the angle of attack from the design space and free from the aerodynamic constraint.

The structural analysis is performed by a structural module. For a given planform geometry, the module InAirSsi generates the internal structural geometry (primary structure).

For each set $\alpha_{\text {geom }}$, the initial structured mesh is deformed and a CFD analysis is perfomed to evaluate $C_{D}$ as well as the maneuver sizing loads. The module InAirSsi generates the structural model from the CFD surface mesh using a random set of primary structure thicknesses $\alpha_{\text {struct }}$ random .

$\alpha_{\text {struct }}$ can be considered as a design parameters and treated at the same level as $\alpha_{\text {geom}}$. In this case the optimization problem will be a highly constrained problem. In this work we choose to treate $\alpha_{\text {struct }}$ in a sub-level. The first reason is to unconstrain the optimization problem. The second reason is deducted by the fact that if 4 planform parameters are enough to impact significantly the aerodynamic objective function, 50 parameters at least are needed to perform a significant sizing of the wing box elements. MGDA is assisted by a metamodel (section II.C) and it is well known that the construction of responces surfaces are limited by the dimension of the design space. Thus, for each $\alpha_{\text {geom }}$ a gradient based algorythm is used to find an optimal set of $\alpha_{\text {struct }}$ that provides internal structure with minimum structural weight that preserves the structural integrety of the wing under 
sizing loads (2.5g). At each step of the gradient-based optimization process, the structural characteristics, the structural wing weight as well as the material stresses aggregated into the Kreisselmeier-Steinhauser REF function and their gradients are computed by the module InAirSsi. The points of the aerostructural database are all computed at the same lift coefficient and a structure that withstand the sizing loads $(\mathrm{KS} \longrightarrow 0)$.

\subsubsection{Results}

MGDA realizes a cooperative optimization of the 2 objectives based on a surrogate models (section II.C), the obtained Pareto set at the iteration $i$ is then evaluated using high fidelity codes and is added to the initial database to generates the surrogate model of the iteration $i+1$.

Fig 17(a) compares the design points when the structural weight is computed using a random wing box thicknesses $\alpha_{\text {struct }}$ random $_{\text {and when the element }}$ thicknesses are sized, this sizing corresponds to a translation of the pareto front. MGDA is applied to an initial database of 20 points Fig 17(a) in a design space of dimension $\in \mathbb{R}^{4}$. The bounds of the design variables are fixed to $[-10 \%,+10 \%]$ for the thickess-to-chord ratio, $\left[-3^{\circ},+3^{\circ}\right]$ for the sweep angle and $[-5 m,+5 m]$ for span modifications.

After 5 steps of MGDA with exact perfomance evaluation, the progression of the first-nondominated set is plotted in Fig 18. The first non-dominated set crosses the Pareto front and this is due to a poor design region for which $C d p \in[140(D C), 150(D C)]$. Was the choice of the initial database dimension enough? Are the bounds of the design space not adapted to this problem? To limit the discussions to the main purpose of the paper, the authors will focuses on the analysis of the two design regions well distinguishable region 
A Fig20(a) and region B Fig20(b).

Two Pareto-optimal configurations that belongs to the region $\mathrm{A}$ and region $\mathrm{B}$ are analysed. Table 2 lists the design variables for both configurations $\operatorname{Design}_{A}$ and $\operatorname{Design}_{B}$. The total near-field drag of Design ${ }_{A}$ is $20.5 \mathrm{DC}$ lower than the total near-field of $\operatorname{Design}_{B}$. A drag decomposition is performed using FFD72 ref, Table 3 lists near-field and far-field drag components. $\operatorname{Design}_{A}$ has a span $2.08 \mathrm{~m}$ longer that $\operatorname{Design}_{B}$ which contributes in reducing the induced drag by 21.96 (DC). The combination of both higher sweep angle for $\operatorname{Design}_{B}$ and a higher thickness-to-chord ratio at section 1 of Design $A$ results in a $\operatorname{Design}_{B}$ that has 1.76 DC less of wave drag than Design . $_{\text {. }}$ The Cp and aerodynamic load distributions of both configurations are plotted in Fig 21. The configuration from region $\mathrm{A}$ is 5.555, 9 tonnes heavier than $\operatorname{Design}_{B}$ to resists the higher aerodynamic loads acting on the structure. 


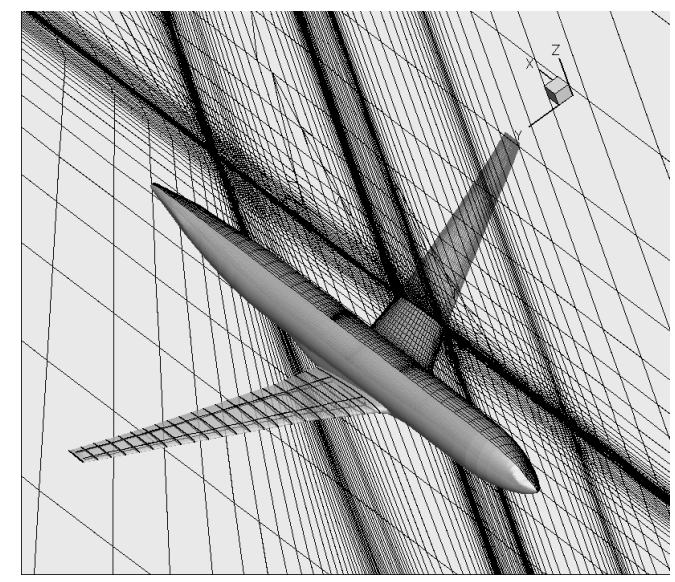

(a) Aerodynamic mesh and the internal structural model of the XRF1 configuration

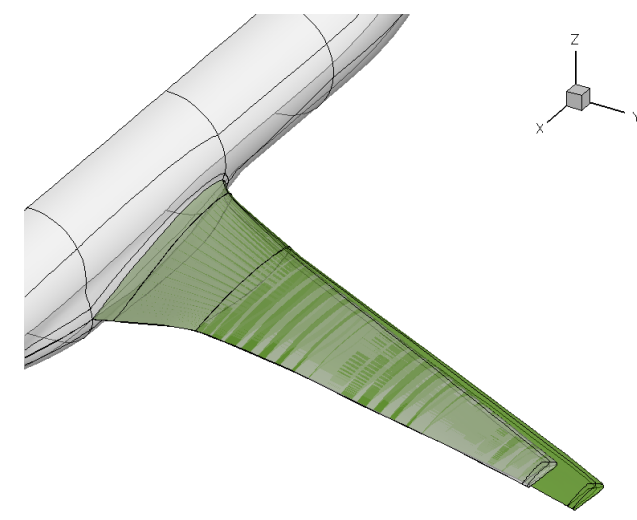

(b) Span and sweep angle modifications

Figure 16: Airbus 3D configuration XRF1

\begin{tabular}{lllll}
\hline \hline & Span & Sweep & Thickness & Thickness \\
& & & 1 & 2 \\
\hline $\operatorname{Design}_{A}$ & 30.04 & $2.95^{\circ}$ & 1.05 & -8.98 \\
$\operatorname{Design}_{B}$ & 27.96 & $5.58^{\circ}$ & -0.012 & -2.64 \\
\hline
\end{tabular}

Table 3: $\alpha_{\text {geom }}$ for $\operatorname{Design}_{A}$ and $\operatorname{Design}_{B}$ 


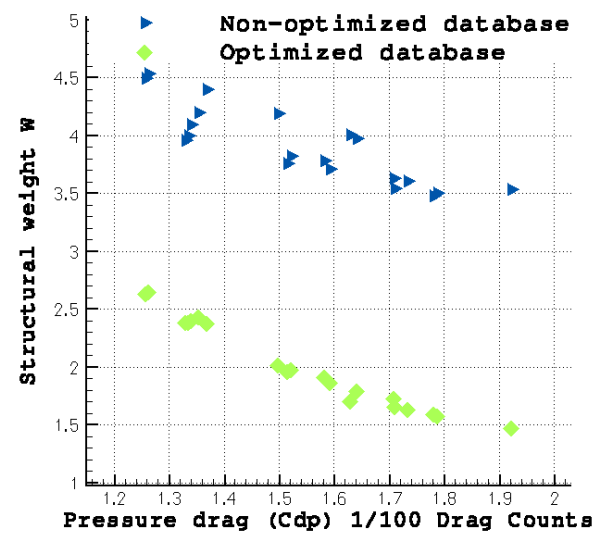

(a) Visualisation of a set of design points for optimal and random $\alpha_{\text {struct }}$

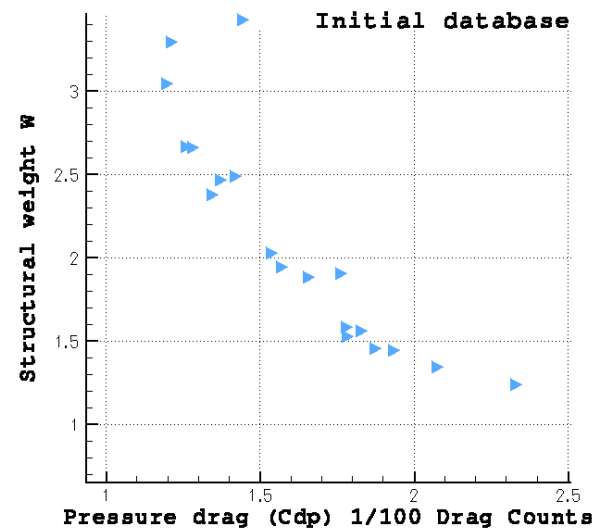

(b) Distribution of initial design points

Figure 17: Aerostructural design space

\begin{tabular}{lllll}
\hline \hline & $\mathrm{CD}_{p}$ & $\mathrm{CD}_{w}$ & $\mathrm{CD}_{i}$ & $\mathrm{~W}$ \\
$\operatorname{Design}_{A}$ & 132.60 & 13.74 & 76.32 & $2,3274.7$ \\
$\operatorname{Design}_{B}$ & 153.13 & 11.97 & 98.28 & $1,7718.8$
\end{tabular}

Table 4: Drag breakdown for $\operatorname{Design}_{A}$ and $\operatorname{Design}_{B}$ 


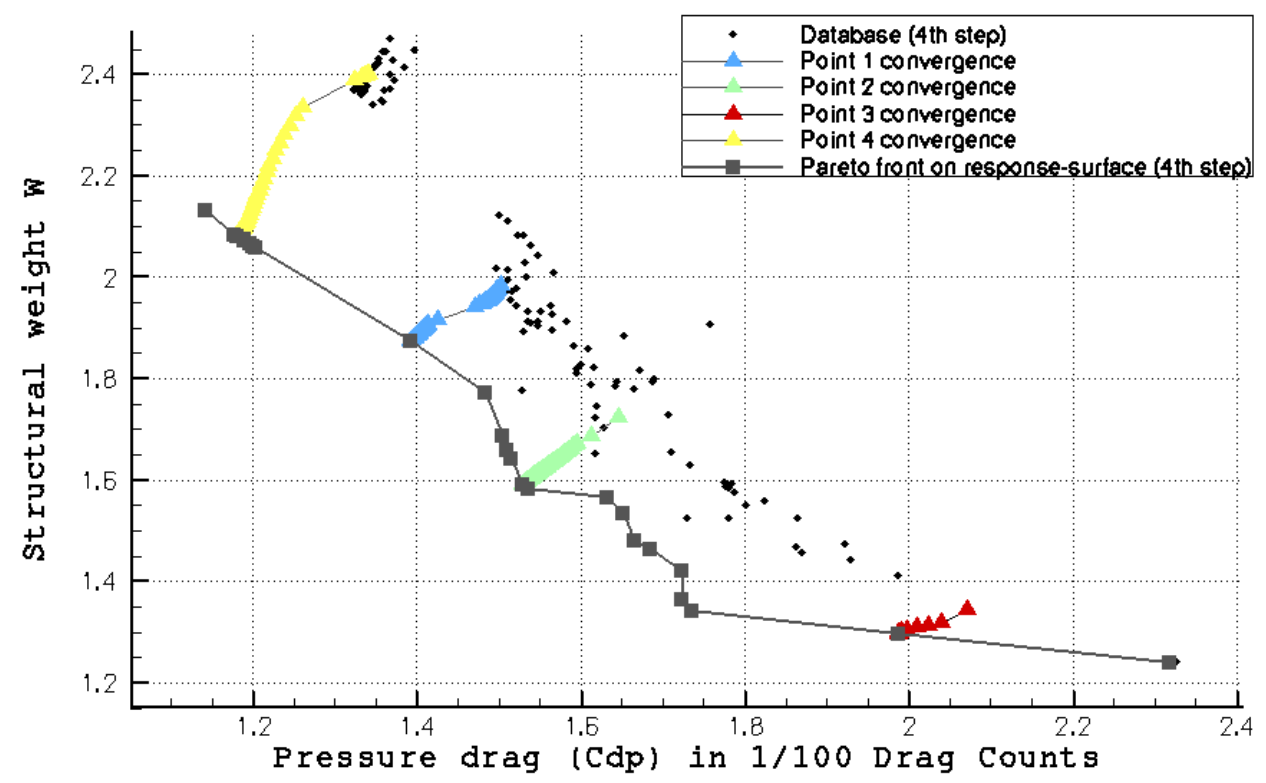

Figure 18: Convergence history at fixed maximum high fidelity function evaluation calls. 


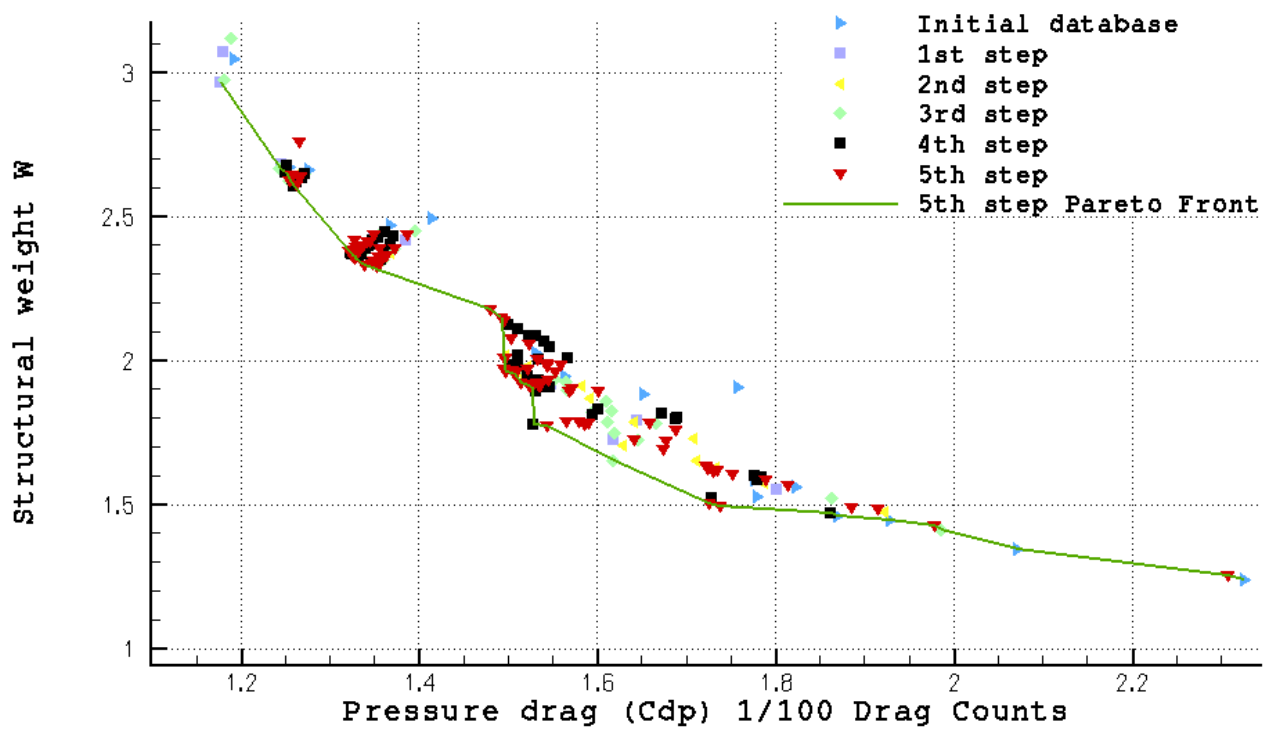

Figure 19: Convergence history of MGDA based on high fidelity for objectives evaluation 


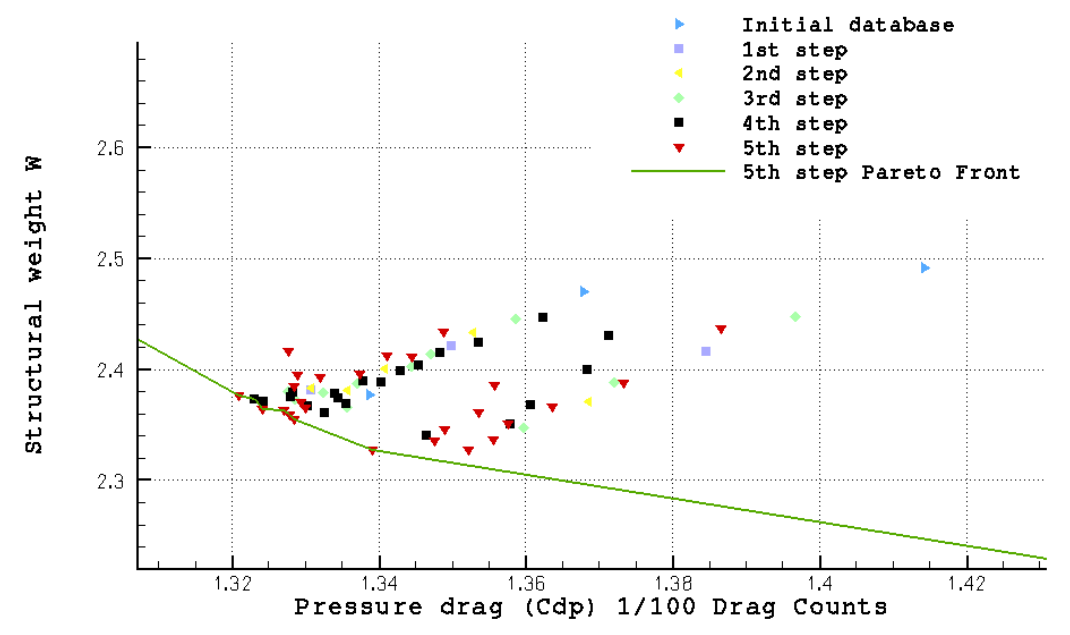

(a) Zoom in region A of the convergence history of MGDA

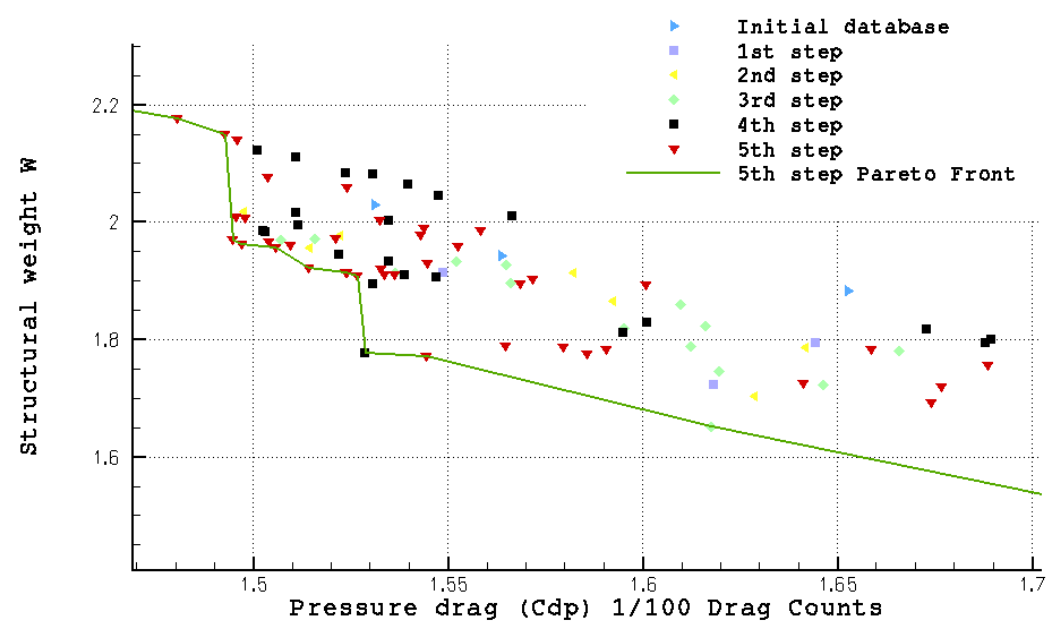

(b) Zoom in region B of the convergence history of MGDA

Figure 20: 


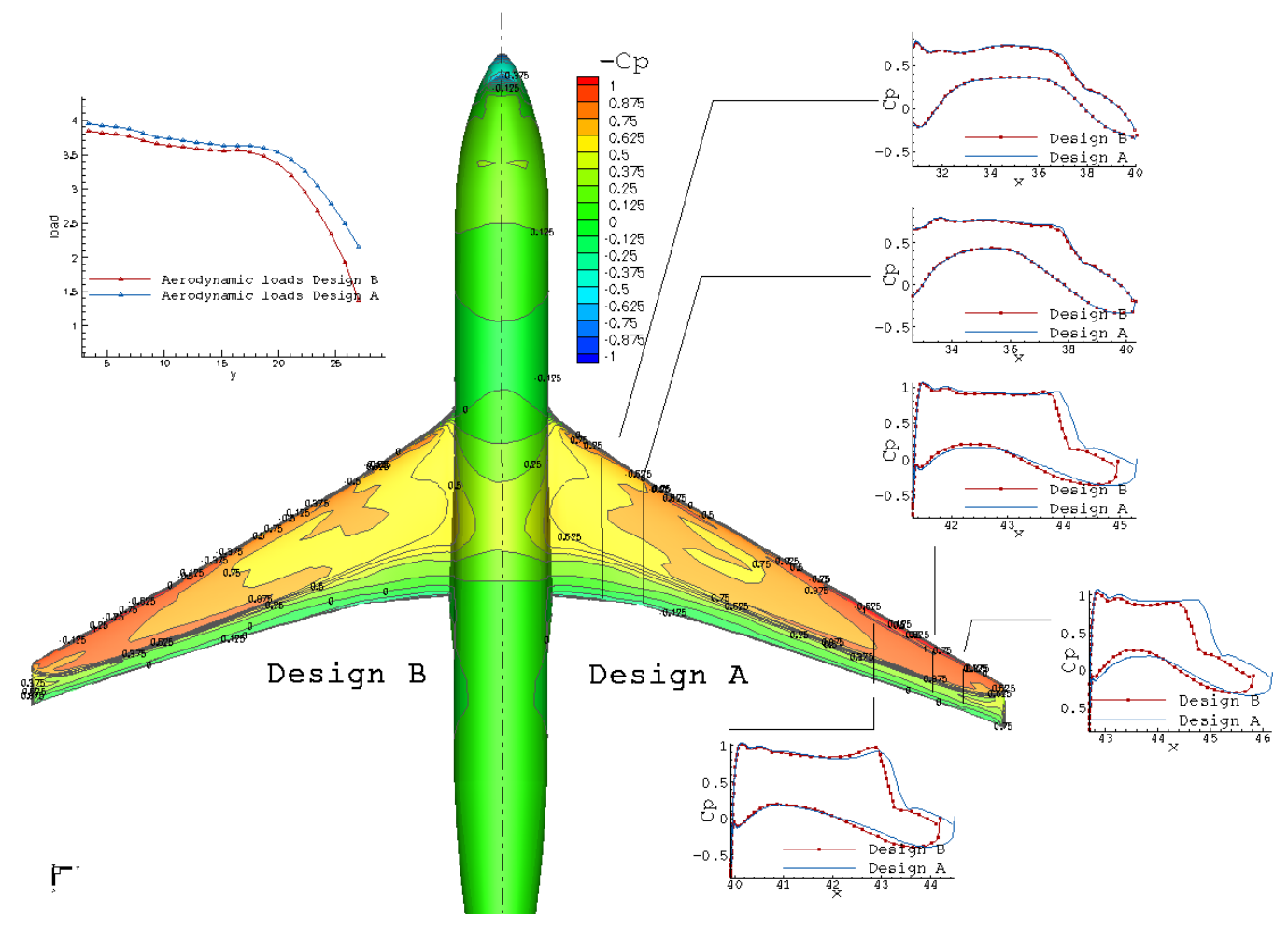

Figure 21: Pressure distribution of selection Pareto-optimal design point 


\section{Conclusions}

In this article, we have first reviewed the basic properties of an iterative method for multi-objective optimization, the Multiple-Gradient Descent Algorithm (MGDA) in which from the knowledge of the local gradients, a direction of search is identified to provide directional derivatives of the objective functions of given sign. This method generalizes the steepest-descent method to the multi-objective context and converges to Pareto-optimal design-points.

A meta-model-assisted variant of the basic method has been proposed for situations in which the gradients are not known exactly or computationally costly to evaluate. The new algorithm is a mixed-fidelity-model approach. It proceeds with the following operations at each cycle (or step):

- calculation of a database of function values by the high-fidelity models, and elaboration of corresponding meta-models;

- convergence by MGDA of the surrogate multi-objective optimization problem associated with the meta-models, initiated from a subset of the meta-models training points;

- database enrichment with the above converged design-points.

We have applied the present method to three multi-objective optimumshape design problems subject to 3D compressible aerodynamics: (i) liftdrag optimization, (ii) drag and sonic-boom reduction, and (iii) drag and structural weight reduction. The surrogate models are improved at each

cycle of the above process. As a result, in all three cases, the capability of the meta-model-assisted method to converge to the Pareto-front associated 
with the high-fidelity models was observed. Typically, less than 10 cycles of the database enrichment are sufficient for a satisfactory description of the front, and this usually results in a significant reduction in computational effort as compared to the requirements of standard evolutionary strategies for multi-objective optimization. 


\section{Acknowledgements}

The authors would like to thank warmly their colleagues G. Carrier, R. Duvigneau and I. Salah El Din for their invaluable suggestions.

This study was partially supported by the French National Research Agency (ANR) Project OMD (Multi-Disciplinary Optimization), Phase 2.

\section{References}

[1] Gill PE, Murray W, Wright MH. 1981. Practical optimization. Academic Press Inc.,Harcourt Brace Jovanovich Publishers, 1981.

[2] Goldberg DE. Genetic Algorithms in Search, Optimization and Machine Learning. Addison-Wesley Longman Publishing Co., 1989.

[3] Deb K, Thiele L, Laumanns L, Zitzler E. Scalable Test Problems for Evolutionary Multi-Objective Optimization. TIK-Technical Report 112, 2001.

[4] Zitzler E, Laumanns M, Thiele L. SPEA2 : Improving the strength Pareto evolutionary algorithm. TIK-Technical Report 103,2001.

[5] Deb K, Pratap A, Agarwal S, Meyarivan T. A fast and elitist multiobjective genetic algorithm : NSGA-II. IEEE transaction on Evolutionary Computation. 2002;6:182-197.

[6] Knowles JD, Corne DW. The Pareto Archived Evolution Strategy : A new baseline algorithm for Pareto multiobjective optimization. In proceedings of Congress of Evolutionary Computation (CEC 99). 1999;1:98105. 
[7] Hadley G. Nonlinear and dynamic programming. Addison-Wesley, 1970.

[8] Désidéri JA. Multiple Gradient Algorithm (MGDA). INRIA, Research Report 6953, Version 3, 2012.

[9] Zerbinati A, Désidéri JA, Duvigneau R. Comparison between MGDA and PAES for multi objective optimization, INRIA Research Report $7667,2011$.

[10] Geuzaine C, Remacle JF. Gmsh:A 3D finite element mesh generator with builtin preand postprocessing facilities. 2009;79(11),1309-1331.

[11] Désidéri JA. Multiple-gradient descent algorithm (MGDA) for multiobjective optimization, Comptes Rendus Mathématique,350(5-6):313-318, 2012.

[12] Désidéri JA MGDA Variants for Multi-Objective Optimization. INRIA, Research Report 8068, Version 1, 2012

[13] National Academy of Science, Commercial Supersonic Technology. The Way Ahead, National Academy Press, 2001.

[14] Welge HR, Nelson C, Bonet J. Supersonic vehicle systems for the 2020 to 2035 timeframe, AIAA paper 2010-4930 2010.

[15] Pierce AD, Maglieri DJ. Effects of atmospheric irregularities on sonicboom propagation, The Journal of the Acoustical Society of America, $1972 ; 51(2 \mathrm{C}), 702-721$.

[16] Cheung SH, Edwards TA, Lawrence SL. Application of CFD to sonic boom near and mid flow-field prediction, NASA TM-102867, 1990. 
[17] Kandil OA, Ozcer IA. Sonic boom computations for double-cone configuration using CFL3D, FUN3D and full-potential codes, AIAA paper 2006-0414, 2006.

[18] Plotkin K, Page J, Extrapolation of Sonic Boom Signatures from CFD Solution, AIAA paper 2002-0922, 2002.

[19] Hayes WD, Haefeli RC, Kulsrud HE. Sonic boom propagation in a stratified atmosphere with computer program, NASA CR-1299, 1969.

[20] Cambier L, Gazaix M. An efficient object-oriented solution to CFD complexity, AIAA paper 02-0108, 2002.

[21] Taylor AD. The TRAPS sonic boom program, NOAA Technical memorandum ERL-ARL-87, 1980.

[22] Sullivan BM, Klos J, Bnehrle RD, McCurdy DA, Haering EA. Human Response to Low-Intensity Sonic Booms Heard Indoors and Outdoors, NASA TM-2010-216685, 2010.

[23] Brezillon J, Carrier G, Laban M. Multi-Disciplinary Optimization Including Environmental Aspects Applied to Supersonic Aircraft, In 7th ICAS conference proceedings 2010.

[24] Dumont A, Ghazlane I, Marcelet M, Carrier C, Salah El Din I, Overview of Recent Development of Aeroelastic Adjoint Method for Civil Aircraft Wing Optimization, in proceedings of ONERA DLR Aerospace Symposium, 2011. 
[25] Ghazlane I, Carrier C, Dumont A, Marcelet M. Aero-Structural Optimization with the Adjoint Method, in proceedings of Eurogen 2011

[26] Brezillon J, Dwight RP, Wild J. Numerical Aerodynamic Optimization of 3D High-lift Configurations, in proceedings of $26^{\text {th }}$ ICAS Congress, 2008.

[27] Martins JRR, Poon NK. On Structural Optimization using Constraint Aggregation, in proceeding of $6^{\text {th }}$ World Congress on Structural and Multidisciplinary Optimization, 2005.

[28] Jameson A, Leoviriyakit K, Shankaran S. Multi-point Aero-structural Optimization of Wings Including Planform Variations, AIAA paper 2007-0000, 2007.

[29] Leoviriyakit K. Wing Planform Optimization via an Adjoint Method, PhD thesis, Stanford University, 2005.

[30] Roux E. Modle de Masse Voilure, PhD thesis, ONERA - Ecole doctoral de SupAro, 2006.

[31] Miettinen K. Nonlinear multiobjective optimization. Vol. 12. Springer, 1999. 